REVISIONES

Rev Obstet Ginecol Venez. 2021; 81 (1): 75-85

https://doi.org/10.51288/00810111

\title{
Neoplasia intraepitelial endometrial: una lesión precursora de cáncer de endometrio
}

\author{
Ernesto Lara. ${ }^{1}$
}

\begin{abstract}
RESUMEN
El cáncer de endometrio representa mundialmente la sexta patología maligna más común en la población femenina, el tipo endometrioide constituye la forma más frecuente, usualmente desarrollado a partir de una secuencia típica de hiperplasia endometrial secundaria a la exposición sostenida a estrógenos sin oposición equilibrada por progestágenos. Se han descrito diferentes sistemas de clasificación para la hiperplasia endometrial, el más reciente, publicado por la Organización Mundial de la Salud en 2014, propone dos categorias: 1) hiperplasia sin atipias y 2) hiperplasia atípica o neoplasia intraepitelial endometrial. Esta clasificación evita la confusión debida a los diferentes términos en uso y refleja un mejor entendimiento del comportamiento de la patología. La hiperplasia atípica o neoplasia intraepitelial endometrial se considera una lesión precursora de carcinoma endometrial tipo I. Los profesionales de la salud deben manejar una terminología estandarizada, diagnosticar con precisión esta entidad y garantizar un tratamiento adecuado de la misma.
\end{abstract}

Palabras clave: Neoplasia intraepitelial endometrial, Hiperplasia endometrial, Hiperplasia endometrial atípica, Cáncer de endometrio.

\section{SUMMARY}

Endometrial cancer represents worldwide the sixth most common malignant pathology in the female population, the endometroid type constitutes the most common form, usually developed from a typical sequence of endometrial hyperplasia secondary to sustained exposure to unopposed estrogens balanced by progestogens. Different classification systems for endometrial hyperplasia have been described, the most recent, published by the World Health Organization in 2014, proposes two categories: 1) hyperplasia without atypia, and 2) atypical hyperplasia or endometrial intraepithelial neoplasia. This classification avoids confusion due to the different terms in use and reflects a better understanding of the pathology behavior. Atypical hyperplasia or endometrial intraepithelial neoplasia is considered a precursor lesion to endometrial carcinoma type I. Health professionals must handle standardized terminology, accurately diagnose this entity, and ensure proper treatment of it.

Keywords: Endometrial intraepithelial neoplasia, Endometrial hyperplasia, Atypical hyperplasia, Endometrial cancer.

\section{INTRODUCCIÓN}

La hiperplasia endometrial (HE) constituye un grupo heterogéneo de lesiones patológicas que van desde proliferaciones reversibles hasta lesiones precursores de cáncer de endometrio (CE), pudiendo definirse, como una proliferación de glándulas endometriales

\footnotetext{
${ }^{1}$ Especialista en Ginecología Oncológica, Servicio de Ginecología. Hospital Dr. Domingo Luciani. Instituto Venezolano de los Seguros Sociales (IVSS). Caracas, Venezuela.
}

de forma y tamaño irregular con un incremento en la relación glándula/estroma, comparado con el endometrio proliferativo (1).

La incidencia de HE incrementa con la edad, con un estimado general de 133 por 100000 mujeres al año, rara vez vista en menores de 30 años, con una mayor incidencia entre 50 y 54 años (2), la edad promedio del paciente con neoplasia intraepitelial endometrial (NIE) es de 53 años $(3,4)$. En 2014, la Organización Mundial de la Salud (OMS), modificó la clasificación 
de 1994, incluyendo solo 2 categorías: 1) hiperplasia sin atipias y 2) hiperplasia atípica (HA) o NIE. Esta reducción a dos categorías se hizo, no solamente para acabar con la multitud de términos confusos en uso, sino para reflejar un mejor entendimiento de los cambios moleculares y genéticos (4), desarrollándose en función de las características histológicas y el potencial oncogénico.

Los factores de riesgo asociados a NIE son los mismos asociados a HE sin atipias y $\mathrm{CE}$ y resultan de la exposición del endometrio sin oposición a estrógenos de fuentes endógenas o exógenas (5). La HE sin atipias raramente progresa a CE $(1 \%-3 \%)$ y no comparte las mismas mutaciones genéticas que su contraparte $(6,7)$, considerándose la NIE la lesión precursora del $\mathrm{CE}$ endometrioide (tipo I) el cual representa entre el $70 \%$ - $80 \%$ de CE (8 - 10). La HA/NIE exhibe muchas de las mutaciones típicas del $\mathrm{CE}$ endometrioide $\mathrm{y}$ hasta en $60 \%$ de los casos los pacientes presentan $\mathrm{CE}$ coexistente o tienen un riesgo extremadamente alto de desarrollar CE, con implicaciones evidentes para el tratamiento, debiendo tratarse generalmente la NIE/ HA mediante histerectomía total (no supracervical), considerando solamente el tratamiento no quirúrgico con control histológico en casos excepcionales (deseos de mantener la fertilidad o presencia de comorbilidades médicas que impidan el tratamiento quirúrgico) (11).

Realizar una distinción adecuada entre hiperplasia endometrial y verdaderas lesiones preinvasoras como la HA/NIE, tiene efectos clínicos significativos, ya que su diferente potencial oncogénico debe coincidir con una intervención terapéutica apropiada evitando el subtratamiento o sobretratamiento.

\section{Sistemas de clasificación de la hiperplasia endometrial}

Se han utilizado múltiples sistemas de clasificación patológica, que se remontan a 1963, para describir HE (12). Antes de 1985, términos como "hiperplasia leve, moderada y severa" a menudo se usaban en los Estados Unidos, mientras que "quístico" e "hiperplasia adenomatosa" se utilizaban más en Europa. Para 1982, la confusión en la terminología y el desacuerdo en los criterios entre expertos, incluso dentro del mismo país, se volvieron más evidentes $(13,14)$.

En 1994, la Organización Mundial de la Salud propuso su clasificación para la hiperplasia endometrial (OMS 94), basada en el estudio realizado por Kurman y cols. (15), en 1985, que correlacionó la presencia de alteraciones citológicas y arquitecturales con el riesgo de desarrollar CE y tuvo el objetivo de estandarizar la terminología en todo el mundo. La clasificación OMS 94 utiliza dos criterios: complejidad glandular y atipia nuclear. Esto dio como resultado cuatro categorías: simple sin atipias, compleja sin atipias, simple atípica e hiperplasia compleja con atipias, las cuales tienen diferentes riesgos de progresión de $1 \%, 3 \%, 8 \%$ y $29 \%$, respectivamente (15). Sin embargo, la interpretación de las características microscópicas esenciales aún era subjetiva y los estudios internacionales posteriores han demostrado, de forma independiente, que la clasificación OMS 94 no es muy reproducible (16 - 18). Además, las categorías individuales no sugieren algoritmos de gestión específicos (19).

Debido a la escasa reproducibilidad de los diagnósticos, en el año 2000, el International Endometrial Collaborative Group, propuso la clasificación de neoplasia intraepitelial endometrial (20), esta se basa en estudios morfológicos, genéticos moleculares, biológicos celulares y pronósticos morfológicos integrados, distinguiéndose tres categorías: 1) hiperplasia benigna (una lesión difusa dependiente de hormonas, que es policlonal). 2) NIE (al principio una proliferación "clonal" localizada, que es monoclonal y neoplásica, que en una etapa avanzada, puede convertirse en una lesión más difusa). 3) Cáncer. El diagnóstico de NIE debe cumplir 5 criterios en un solo fragmento, que incluyen el apiñamiento de la glándula 
Cuadro 1. Definición de neoplasia intraepitelial endometrial. Criterios $(20,21)$

\begin{tabular}{ll}
\hline Criterios NIE & Comentarios \\
\hline Arquitectura & $\begin{array}{l}\text { El área de las glándulas excede a la } \\
\text { del estroma }\end{array}$ \\
Citología & $\begin{array}{l}\text { La citología difiere entre el foco } \\
\text { arquitectónicamente lleno y el } \\
\text { fondo }\end{array}$ \\
Diámetro $>1 \mathrm{~mm}$ & $\begin{array}{l}\text { La dimensión lineal máxima de la } \\
\text { lesión excede 1 mm } \\
\text { Condiciones benignas con criterios }\end{array}$ \\
Excluir imitadores & $\begin{array}{l}\text { superpuestos: basal, secretorio, } \\
\text { pólipos, reparación, etc. } \\
\text { Carcinoma si hay glándulas } \\
\text { serpenteantes como laberintos, áreas } \\
\text { sólidas o cribiformes apreciables }\end{array}$ \\
\hline
\end{tabular}

arquitectónica, la citología alterada, el tamaño mínimo de $1 \mathrm{~mm}$, la exclusión de carcinoma y la exclusión de imitadores (Cuadro 1).

Para el año 2003, la OMS propone el sistema NIE como una alternativa a la clasificación de 1994, con el propósito de mejorar la predicción de los resultados clínicos, mejorar la reproducibilidad entre observadores y reducir el sesgo subjetivo inherente a la clasificación de la OMS, de 1994 (22). En el 2104 es cuando se publica una nueva clasificación de la HE, que consta de 2 categorías: 1) hiperplasia sin atipias 2) hiperplasia atípica/neoplasia intraepitelial endometrial. Esta reducción a dos categorías se debió a la necesidad de eliminar la multitud de términos confusos en uso y reflejar una nueva comprensión de los cambios genéticos y moleculares (5).

La hiperplasia sin atipia no exhibe cambios genéticos relevantes, son cambios benignos y desaparecerán después de que el medio endocrino (niveles fisiológicos de progesterona) se haya normalizado. En algunos casos (1\% - $3 \%$ ), puede ocurrir progresión a enfermedad invasiva si el trastorno endocrino (predominio de estrógenos a largo plazo o deficiencia de progesterona absoluta o relativa) persiste a largo plazo. El término HA/NIE presenta muchas de las mutaciones típicas del CE tipo endometrioide (23). Hasta en el $60 \%$ de los casos, pueden coexistir con CE o presentan un riesgo extremadamente alto de desarrollar patología invasora (5) (Cuadro 2).

Cuadro 2. Clasificación de la hiperplasia endometrial, OMS 2014 (11)

\begin{tabular}{|c|c|c|c|c|}
\hline Término & Sinónimos & Cambios genéticos & $\begin{array}{l}\text { Carcinoma } \\
\text { endometrial } \\
\text { coexistente }\end{array}$ & $\begin{array}{l}\text { Progresión } \\
\text { a carcinoma } \\
\text { endometrial }\end{array}$ \\
\hline $\begin{array}{l}\text { Hiperplasia } \\
\text { sin atipia }\end{array}$ & $\begin{array}{l}\text { Hiperplasia benigna endometrial, } \\
\text { hiperplasia endometrial simple no } \\
\text { atípica; hiperplasia endometrial no } \\
\text { atípica compleja; hiperplasia endometrial } \\
\text { simple sin atipia; hiperplasia endometrial } \\
\text { compleja sin atipia }\end{array}$ & $\begin{array}{l}\text { Bajo nivel de mutaciones } \\
\text { somáticas en glándulas dispersas } \\
\text { con morfología en la tinción HE } \\
\text { que no muestra cambios }\end{array}$ & $<1 \%$ & $\begin{array}{c}\text { RR: } \\
1,01-1,03\end{array}$ \\
\hline $\begin{array}{l}\text { Hiperplasia } \\
\text { atípica/ } \\
\text { neoplasia } \\
\text { intrepitelial } \\
\text { endometrial }\end{array}$ & $\begin{array}{l}\text { Hiperplasia endometrial compleja con } \\
\text { atipias, hiperplasia endometrial simple } \\
\text { con atipia, neoplasia intraepitelial } \\
\text { endometrial }\end{array}$ & $\begin{array}{l}\text { Muchos de los cambios genéticos } \\
\text { típicos para el cáncer de } \\
\text { endometrio endometrioide pueden } \\
\text { estar presentes, incluyendo: } \\
\text { inestabilidad de microsatélites; } \\
\text { inactivación de PAX2; mutación } \\
\text { de PTEN, KRAS y CTNNB1 } \\
(\beta \text {-catenina) }\end{array}$ & $\begin{array}{c}25 \%-33 \% \\
59 \%\end{array}$ & $\begin{array}{c}\text { RR: } \\
14-45\end{array}$ \\
\hline
\end{tabular}


El diagnóstico diferencial entre las lesiones uterinas benignas y la HA/NIE se basa principalmente en criterios morfológicos, pero puede estar respaldado por marcadores inmunohistoquímicos adicionales y alteraciones moleculares en algunos casos (21).

El dictamen del comité de The American College of Obstetricians and Gynecologists (ACOG) y Society of Gynecologic Oncology (SGO) recomienda el uso del esquema NIE para una terminología más clara y distinguir las lesiones premalignas (19). En 2016, se publicaron las directrices conjuntas de dos comités: el Royal College of Obstetricians and Gynecologists (RCOG) y British Society for Gynecological Endoscopy (BSGE), sobre el tratamiento y la clasificación de la hiperplasia (24), recomendaron la clasificación OMS 2014, que divide la HE en dos grupos: hiperplasia sin atipia e HA/NIE. Igualmente, en 2019 la Society of Gynecologic Oncology of Canada (GOC) y la Society of Obstetricians and Gynaecologists of Canada (SOGC) (25), publicaron una guía para la clasificación y el manejo de la HE, adoptando la clasificación propuesta por la OMS en 2014.

\section{Herramientas diagnósticas para NIE}

La HE a menudo se sospecha en mujeres que presentan sangrado uterino anormal. Sin embargo, la confirmación del diagnóstico requiere un análisis histológico de las muestras de tejido endometrial obtenidas mediante el uso de dispositivos de succión ambulatorios en miniatura, diseñados para raspar y/o aspirar ciegamente el tejido endometrial de la cavidad uterina, o mediante toma de muestras endometriales para pacientes hospitalizadas, a través de la dilatación y curetaje (DC), realizados bajo anestesia general. La toma de muestra endometrial también es fundamental para controlar la regresión, la persistencia o la progresión (24).

La detección sensible y específica de HA/NIE y la exclusión de carcinoma coexistente son prerrequisitos para el manejo de pacientes con lesiones endometriales premalignas. Excluir carcinoma concurrente por biopsia endometrial ambulatoria es especialmente problemático: aproximadamente el $40 \%$ de las pacientes que reciben un diagnóstico de NIE de esta manera, presentan un diagnóstico de carcinoma en el espécimen de histerectomía $(26,27)$. La precisión de la DC, en comparación con los dispositivos de aspiración endometrial en el diagnóstico de precáncery excluyendo el carcinoma concurrente, no está clara. Ambos tienen limitaciones de muestreo: aproximadamente el $60 \%$ de las muestras de DC muestrean menos de la mitad de la cavidad uterina (28), el método de muestreo es menos importante si este incluye el tratamiento definitivo con una histerectomía, lo que elimina el riesgo de no diagnosticar $\mathrm{CE}$. Se ha informado que los métodos para toma de muestra con dispositivos de succión endometrial y DC producen tasas iguales de detección de cáncer en pacientes con sangrado uterino anormal. Una serie retrospectiva de una sola institución encontró que la DC utilizada para el diagnóstico de NIE era menos probable que fallara en no diagnosticar cáncer (que fue evidente en la histerectomía posterior) que el uso de dispositivo de succión endometrial (27\% en comparación con $46 \%$, respectivamente) (29). Estos métodos son considerados "enfoques ciegos" y usualmente muestrean menos del $50 \%$ de la cavidad endometrial. En ciertas circunstancias pueden fallar en proveer una suficiente cantidad de tejido y por lo tanto impedir un adecuado diagnóstico. En tal escenario, métodos alternativos o adicionales para muestrear el endometrio serán necesarios. Una paciente sin un diagnóstico definitivo ante una alta sospecha de HE o CE, sangrado persistente, biopsias de endometrio fallidas, se le debería ofrecer una estrategia diagnóstica alternativa (25). El diagnóstico histeroscópico mediante biopsia dirigida y legrado son recomendados en estas situaciones para incluir tanto una lesión focal como todo el endometrio (19).

El ultrasonido transvaginal (USTV) tiene un excelente valor predictivo negativo para $\mathrm{CE}$ en mujeres con sangrado posmenopáusico. Cuando es realizado en 
mujeres con sangrado posmenopáusico y el espesor endometrial es $4 \mathrm{~mm}$ o menos, la biopsia de tejido endometrial no es necesaria debido al muy bajo riesgo de malignidad uterina en estas pacientes. Un grosor endometrial mayor a $4 \mathrm{~mm}$ en paciente con sangrado posmenopáusico debe ameritar una evaluación alternativa (biopsia endometrial, histeroscopia o sonohisterografia). Una medición endometrial mayor a $4 \mathrm{~mm}$ que se descubre de manera accidental en una paciente posmenopáusica sin sangrado no necesita desencadenar otra evaluación de forma rutinaria, aunque es apropiada una evaluación individualizada basada en las características de la paciente y los factores de riesgo (30).

\section{Manejo de la neoplasia intraepitelial endometrial}

La NIE tiene un alto riesgo de progresión a CE y un potencial de enfermedad invasiva concurrente (27, 31). Estudios observacionales han reportado que el carcinoma subyacente es encontrado en hasta el $60 \%$ de los especímenes reportados con NIE (27, 32-36). Por ello, la histerectomía es el tratamiento de elección para la mayoría de las mujeres con HA/NIE. La terapia con progestágenos es una opción razonable para las mujeres que desean preservar la fertilidad o que no pueden tolerar la cirugía (25).

\section{Manejo quirúrgico}

La histerectomía total es el procedimiento de elección, ya que proporciona una evaluación definitiva de un posible carcinoma concurrente y trata eficazmente la lesión premaligna. Actualmente, las opciones quirúrgicas incluyen procedimientos abdominales, vaginales y mínimamente invasivos (como el abordaje laparoscópico o robótico) (37). Estos métodos son aceptables para realizar una histerectomía con o sin salpingooforectomia bilateral en pacientes con diagnóstico de NIE. La salpingooforectomíabilateral no es absolutamente necesaria, especialmente en mujeres premenopáusicas y, de hecho, extirpar ambos ovarios en mujeres premenopáusicas o perimenopáusicas sin un diagnóstico de cáncer ginecológico puede resultar en un aumento de la morbilidad y mortalidad en general.

El enfoque quirúrgico depende de la extensión del procedimiento planificado y la habilidad del cirujano. Los estudios clínicos indican que, en las manos correctas, la histerectomía laparoscópica total, la histerectomía asistida por robot o la histerectomía vaginal se asocian con menos dolor, alta hospitalaria más temprana y recuperación más rápida en comparación con la histerectomía abdominal $(38,39)$.

La histerectomía supracervical, morcelación y ablación endometrial, son tratamientos inaceptables para la NIE. Debido al riesgo de carcinoma subyacente, no se debe realizar una histerectomía supracervical; la extracción del cuello uterino y el segmento uterino inferior junto con el cuerpo uterino permite la determinación de cualquier tipo de cáncer descubierto de forma incidental y reduce el riesgo de dejar enfermedad residual (40).

La literatura sobre la precisión del corte congelado en el momento de la histerectomía para descartar cáncer es inconsistente y se limita a pequeños estudios de observación (41 - 47). Varios informes favorecen el corte congelado para determinar qué pacientes requieren estadificación quirúrgica (43 - 47). Una limitación de este enfoque es el potencial de resultados incongruentes entre el corte congelado y el diagnóstico final, lo que podría resultar en sobretratamiento o subtratamiento en el momento de la cirugía (42). Además, existen centros de salud que no cuentan con patólogos para el análisis del corte congelado o un profesional calificado disponible para realizar una linfadenectomía si fuera necesario. Si el carcinoma endometrial se diagnostica finalmente después de la histerectomía, a menudo está bien diferenciado y no 
está asociado con características de alto riesgo que requieren estadificación quirúrgica adicional $(27,41$, 48 ), por lo cual esta cirugía puede llevarse a cabo en centros donde el corte congelado no esté disponible (41). En general, no hay pruebas suficientes para apoyar firmemente el uso rutinario del corte congelado en el manejo de estas pacientes.

La linfadenectomía en el momento de la cirugía para NIE resultaría en un sobretratamiento y un mayor riesgo quirúrgico para la gran mayoría de las pacientes. El CE asociado con NIE diagnosticado en una muestra de histerectomía suele ser una lesión de bajo grado en estadio temprano que tiene un bajo riesgo de diseminación linfovascular. El riesgo de un carcinoma uterino de alto riesgo concurrente en mujeres con un diagnóstico de biopsia de HA/NIE varía entre el $5 \%$ y el $7 \%$ (49 - 51). Por lo tanto, realizar la linfadenectomía pélvica y paraaórtica como parte del tratamiento de la NIE daría lugar a una gran mayoría de pacientes que serían sometidos a riegos asociados a la cirugía estadificadora. La histerectomía total, con o sin ooforectomía, junto con el lavado peritoneal, es el tratamiento quirúrgico más apropiado para estas pacientes (19). La evaluación intraoperatoria del ganglio centinela es una alternativa atractiva, pero actualmente debe considerarse en investigación (37).

Después de la histerectomía total, si no hay CE en la muestra, no es necesaria una vigilancia adicional relacionada con HE. La citología de cúpula vaginal debe realizarse solo si hay una indicación basada en el historial de neoplasia intraepitelial cervical de la paciente $\mathrm{u}$ otras afecciones, no en el contexto de $\mathrm{HE}$ (52).

\section{Manejo no quirúrgico}

La preservación uterina puede ser considerada en pacientes que deseen mantener la fertilidad o en quienes presenten comorbilidades que impidan el tratamiento quirúrgico. El asesoramiento es importante y debería incluir no solo el riesgo de CE subyacente sino el riesgo de CE mayor a estadio I ( $2 \%)$, cáncer de ovario coexistente (4\%) y muerte $(0,5 \%)(53)$. Los objetivos terapéuticos en pacientes que deseen preservar la fertilidad incluyen la eliminación completa de la enfermedad, la reversión a la función endometrial normal y la prevención del CE; en las pacientes que no son candidatas a cirugía por comorbilidad asociada, los objetivos del tratamiento son estabilizar la enfermedad y prevenir el cáncer (37).

Varios estudios han tratado de manipular la naturaleza hormonal de la hiperplasia y el CE dirigiéndose a los receptores hormonales expresados en las lesiones para iniciar la muerte de las células tumorales. Del mismo modo, los estudios también han utilizado objetivos hormonales para revertir las lesiones hiperplásicas o precancerosas. Las clases hormonales con potencial, tanto en la práctica como en la teoría, incluyen progestágenos, moduladores selectivos de los receptores de estrógenos, inhibidores de aromatasa y antagonistas de la hormona liberadora de gonadotropina. La terapia hormonal con derivados de progesterona es de gran interés porque tiene un perfil de toxicidad aceptable, siendo una opción para cualquier paciente que desee mantener la fertilidad y, ciertamente, una consideración para la mayoría de las pacientes de edad avanzada con comorbilidades que llevan el diagnóstico de HA/ NIE (37); la progesterona contraresta los efectos mitogénicos de los estrógenos e induce la diferenciación secretora.

Para las mujeres tratadas con progestágenos para NIE, el tratamiento de primera línea es el sistema intrauterino liberador de levonorgestrel (SILL) y los progestágenos orales como la segunda mejor alternativa (24). El SILL parece ser el tratamiento de progestágenos más efectivo, es fácil de cumplir, bien tolerado y proporciona anticoncepción. El SILL está asociado con tasas de regresión al endometrio normal de aproximadamente 
Cuadro 3. Progestágenos utilizados en el tratamiento conservador de la hiperplasia endometrial (25)

\begin{tabular}{lccc}
\hline Progestágeno & Esquema & $\begin{array}{c}\text { Nombre } \\
\text { comercial }\end{array}$ & Dosis \\
\hline Acetato de medroxiprogesterona & Oral continuo dosis alta & Provera & $100-200 \mathrm{mg} /$ día \\
& Dosis baja & & $2,5-20 \mathrm{mg} / \mathrm{día}$ \\
& Oral cíclico & & $10-20 \mathrm{mg} /$ día \\
& Inyectable & Depo-provera & 10 -12 día/ciclo \\
& Dosis alta & Megace & $80-320 \mathrm{mg} /$ día \\
Acetato de megestrol & Dosis baja & & $40 \mathrm{mg} /$ día \\
Acetato de norestrinona & Oral continuo & Norlutate & $5-12 \mathrm{mg} /$ día \\
Progesterona micronizada & Oral cíclico & & $15 \mathrm{mg} / \mathrm{día}$ \\
Sistema intrauterino liberador de levonorgestrel & & & x 10-12 día/ciclo \\
\hline
\end{tabular}

$90 \%$ (54). El acetato de megestrol es típicamente el progestágeno oral utilizado para HA/NIE porque es más potente que el acetato de medroxiprogesterona $(55,56)$. La metformina también se puede agregar para aumentar el efecto del tratamiento incluso en ausencia de síndrome metabólico $(57,58)$.

El modo y la duración del tratamiento con progestágenos son esenciales para su éxito. Los diferentes tipos de progestágenos y sus dosis en el tratamiento de $\mathrm{HE}$ se presentan en el cuadro 3. La mediana de tiempo para la regresión de HA/NIE al endometrio normal en tratamiento con progesterona parece ser de seis a nueve meses (55, 56, 59), recomendándose realizar el seguimiento cada 3 meses hasta obtener al menos 2 biopsias negativas $(53,59)$. El mayor riesgo de recurrencia se presenta en los 2 primeros años posteriores al cese del tratamiento, por lo que se recomienda biopsia endometrial cada 6 meses por 2 años y posteriormente anual, hasta que los factores de riesgo sean corregidos o una histerectomía total sea realizada $(60,61)$. Las pacientes que experimenten progresión a carcinoma durante el seguimiento, aquellas sin regresión de la hiperplasia después de 12 meses de tratamiento médico o recaída después de completar el tratamiento con progestágenos, quienes continúen experimentando sangrado uterino anormal a pesar del tratamiento o rechacen el seguimiento o tratamiento médico, deberían ser sometidas a tratamiento quirúrgico definitivo (24). En pacientes que no sean candidatas a cirugía, las recurrencias pueden ser tratadas con una segunda ronda de progestágenos con una tasa de respuesta esperada que alcanza hasta el $85 \%$ y una posibilidad de tercera ronda de tratamiento en caso de una segunda recurrencia (62).

\section{DISCUSIÓN}

La NIE es de importancia clínica porque a menudo es una entidad precursora de $\mathrm{CE}$, por lo cual un 
diagnóstico preciso de esta lesión premaligna puede reducir la probabilidad de desarrollar una patología invasora de endometrio. La clasificación de la OMS 2014 refleja un mejor entendimiento de la HE, sobre la base de las características histológicas y en el potencial oncogénico, y representa una importante simplificación en la práctica clínica, particularmente en la elección del tratamiento. La histerectomía total es el tratamiento de elección debido al riesgo de coexistencia y progresión a $\mathrm{CE}$, se considera inaceptable la histerectomía subtotal, la morcelación uterina y la ablación endometrial. El tratamiento no quirúrgico con progesterona puede proporcionar una alternativa a la histerectomía en pacientes que deseen preservar la fertilidad o quienes presenten comorbilidad asociada que contraindique la cirugía.

\section{REFERENCIAS}

1. Hedrick Ellenson L, Ronnett BM, Kurman RJ. Precursor Lesions of Endometrial Carcinoma. In: Kurman RJ, Hedrick Ellenson L, Ronnett, BM, editores. Blaustein's Pathology of the Female Genital Tract. 6a ed. New York: Springer; 2010. p.360-361.

2. Reed SD, Newton KM, Clinton WL, Epplein M, Garcia R, Allison K, et al. Incidence of endometrial hyperplasia. Am J Obstet Gynecol. 2009; 200(6):678. e1-6. doi: 10.1016/j.ajog.2009.02.032. Epub 2009 Apr 23. PMID: 19393600; PMCID: PMC2692753.

3. Ries LAG, Melbert D, Krapcho M, Stinchcomb DG, Howlader N, Horner MJ, et al., editors. SEER Cancer Statistics Review, 1975-2005 [Internet]. Bethesda: National Cancer Institute. MD; 2013 [consultado 23 de abril de 2020] Disponible en: https://seer.cancer.gov/ csr/1975_2005

4. Semere LG, Ko E, Johnson NR, Vitonis AF, Phang LJ, Cramer DW, et al. Endometrial intraepithelial neoplasia: clinical correlates and outcomes. Obstet Gynecol. 2011; 118(1):21-28. doi: 10.1097/AOG.0b013e31821d78af. PMID: 21691159; PMCID: PMC3155976.

5. Zaino R, Carinelli SG, Ellenson LH. Tumours of uterine corpus: epithelial tumours and precursors. En: Kurman RJ, Carcangiu ML, Herrington CS, Young RH, editors. WHO Classification of tumours of female reproductive organs. 4a ed. Lyon: WHO Press; 2014. p.125-134.

6. Abu Hashim H, Ghayaty E, El Rakhawy M. Levonorgestrel-releasing intrauterine system vs oral progestins for non-atypical endometrial hyperplasia: a systematic review and metaanalysis of randomized trials. Am J Obstet Gynecol. 2015; 213(4):469-478. doi: 10.1016/j.ajog.2015.03.037. Epub 2015 Mar 19. PMID: 25797236.

7. Antonsen SL, Ulrich L, Høgdall C. Patients with atypical hyperplasia of the endometrium should be treated in oncological centers. Gynecol Oncol. 2012; 125(1):124-128. doi: 10.1016/j.ygyno.2011.12.436. Epub 2011 Dec 20. PMID: 22198048.

8. Sherman ME. Theories of endometrial carcinogenesis: a multidisciplinary approach. Mod Pathol. 2000 Mar;13(3):295-308. doi: 10.1038/modpathol.3880051. PMID: 10757340.

9. Silverg SG, Kurman RJ, Nogales F, Mutter GL, KubikHuch RA, Tavassoli FA. Epithelial tumours and related lesions. In: Tavassoli FA, Devilee P, editors. Pathology and genetics of tumours of the breast and female genital organs. World Health Organization classification of tumours. Lyon: IARC press; 2003. p.221-32.

10. Setiawan VW, Yang HP, Pike MC, McCann SE, Yu H, Xiang YB, et al. Type I and II endometrial cancers: have they different risk factors? J Clin Oncol. 2013; 31(20):2607-18. doi: 10.1200/JCO.2012.48.2596. Epub 2013 Jun 3. PMID: 23733771; PMCID: PMC3699726.

11. Emons G, Beckmann MW, Schmidt D, Mallmann P; Uterus commission of the Gynecological Oncology Working Group (AGO). New WHO Classification of Endometrial Hyperplasias. Geburtshilfe Frauenheilkd. 2015; 75(2):135-136. doi: 10.1055/s-0034-1396256. PMID: 25797956; PMCID: PMC4361167.

12. Chandra V, Kim JJ, Benbrook DM, Dwivedi A, Rai R. Therapeutic options for management of endometrial hyperplasia. J Gynecol Oncol. 2016; 27(1):e8. doi: 10.3802/jgo.2016.27.e8. Epub 2015 Dec 1. PMID: 26463434; PMCID: PMC4695458.

13. Fox H, Buckley $\mathrm{CH}$. The endometrial hyperplasias and their relationship to endometrial neoplasia. Histopathology. 1982; 6(5):493-510. doi: 10.1111/ j.1365-2559.1982.tb02747.x. PMID: 6754573.

14. Winkler B, Alvarez S, Richart RM, Crum C. Pitfalls in the diagnosis of endometrial neoplasia. Obstet Gynecol. 1984; 64(2): 185-94.

15. Kurman RJ, Kaminski PF, Norris HJ. The behavior of endometrial hyperplasia. A longterm study of "untreated" hyperplasia in 170 patients. Cancer. 1985 Jul 15;56(2):403-12. doi: 10.1002/1097-0142(19850715)56:2<403::aidcncr2820560233>3.0.co;2-x. PMID: 4005805. 
16. Skov BG, Broholm H, Engel U, Franzmann MB, Nielsen AL, Lauritzen AF, et al. Comparison of the reproducibility of the WHO classifications of 1975 and 1994 of endometrial hyperplasia. Int J Gynecol Pathol. 1997; 16(1):33-7. doi: 10.1097/00004347-19970100000006. PMID: 8986530.

17. Kendall BS, Ronnett BM, Isacson C, Cho KR, Hedrick L, Diener-West M, et al. Reproducibility of the diagnosis of endometrial hyperplasia, atypical hyperplasia, and well-differentiated carcinoma. Am J Surg Pathol. 1998; 22(8):1012-1109. doi: 10.1097/00000478-19980800000012. PMID: 9706982.

18. Bergeron C, Nogales FF, Masseroli M, Abeler V, Duvillard P, Müller-Holzner E, et al. A multicentric European study testing the reproducibility of the WHO classification of endometrial hyperplasia with a proposal of a simplified working classification for biopsy and curettage specimens. Am J Surg Pathol. 1999; 23(9):1102-1108. doi: 10.1097/00000478199909000-00014. PMID: 10478671.

19. The American College of Obstetricians and Gynecologists Committee Opinion no. 631. Endometrial intraepithelial neoplasia. Obstet Gynecol. 2015 May;125(5):1272-1278. doi: 10.1097/01. AOG.0000465189.50026.20. PMID: 25932867.

20. Mutter GL. Endometrial intraepithelial neoplasia (EIN): will it bring order to chaos? The Endometrial Collaborative Group. Gynecol Oncol. 2000; 76(3):287290. doi: 10.1006/gyno.1999.5580. PMID: 10684697.

21. Baak JP, Mutter GL, Robboy S, van Diest PJ, Uyterlinde AM, Orbo A, et al. The molecular genetics and morphometry-based endometrial intraepithelial neoplasia classification system predicts disease progression in endometrial hyperplasia more accurately than the 1994 World Health Organization classification system. Cancer. 2005; 103(11):2304-2312. doi: 10.1002/cncr.21058. PMID: 15856484; PMCID: PMC2600877

22. Mutter GL, Bergeron C, Deligdisch L, Ferenczy A, Glant M, Merino M, et al. The spectrum of endometrial pathology induced by progesterone receptor modulators. Mod Pathol. 2008; 21(5):591-598. doi: 10.1038/modpathol.2008.19. Epub 2008 Feb 8. PMID: 18246050 .

23. Cancer Genome Atlas Research Network, Kandoth C, Schultz N, Cherniack AD, Akbani R, Liu Y, Shen $\mathrm{H}$, et al. Integrated genomic characterization of endometrial carcinoma. Nature. 2013; 497(7447):6773. doi: 10.1038/nature12113. Erratum in: Nature.
2013; 500(7461):242. PMID: 23636398; PMCID: PMC3704730.

24. Gallos ID, Alazzam M, Clark TJ, Faraj R, Rosenthal AN, Smith PP, et al., editores. Management of Endometrial Hyperplasia [Internet]. Londres: RCOG/ BSGE Green-top Guideline No. 67; 2016 [consultado 23 de abril de 2020]. Disponible en: https://www.rcog. org.uk/globalassets/documents/guidelines/green-topguidelines/gtg_67_endometrial_hyperplasia.pdf

25. Auclair MH, Yong PJ, Salvador S, Thurston J, Colgan TTJ, Sebastianelli A. Guideline No. 392-Classification and Management of Endometrial Hyperplasia. J Obstet Gynaecol Can. 2019; 41(12):1789-1800. doi: 10.1016/j. jogc.2019.03.025. Erratum in: J Obstet Gynaecol Can. 2020; 42(10):1287. PMID: 31785798.

26. Mutter GL, Kauderer J, Baak JP, Alberts D; Gynecologic Oncology Group. Biopsy histomorphometry predicts uterine myoinvasion by endometrial carcinoma: a Gynecologic Oncology Group study. Hum Pathol. 2008; 39(6):866-874. doi: 10.1016/j.humpath.2007.09.023. Epub 2008 Apr 23. PMID: 18436277; PMCID: PMC2601480.

27. Trimble CL, Kauderer J, Zaino R, Silverberg S, Lim PC, Burke JJ 2nd, et al. Concurrent endometrial carcinoma in women with a biopsy diagnosis of atypical endometrial hyperplasia: a Gynecologic Oncology Group study. Cancer. 2006; 106(4):812-819. doi: 10.1002/cncr.21650. PMID: 16400639.

28. Stock RJ, Kanbour A. Prehysterectomy curettage. Obstet Gynecol. 1975; 45(5):537-541.

29. Leitao MM Jr, Han G, Lee LX, Abu-Rustum NR, Brown CL, Chi DS, et al. Complex atypical hyperplasia of the uterus: characteristics and prediction of underlying carcinoma risk. Am J Obstet Gynecol. 2010; 203(4):349. e1-6. doi: 10.1016/j.ajog.2010.05.004. Epub 2010 Jun 23. PMID: 20576254.

30. ACOG Committee Opinion No. 440: The Role of Transvaginal Ultrasonography in the Evaluation of Postmenopausal Bleeding. Obstet Gynecol. 2009; 114(2 Pt 1):409-411. doi: 10.1097/AOG.0b013e3181b48feb. PMID: 19623006.

31. Lacey JV Jr, Sherman ME, Rush BB, Ronnett BM, Ioffe $\mathrm{OB}$, Duggan MA, et al. Absolute risk of endometrial carcinoma during 20-year follow-up among women with endometrial hyperplasia. J Clin Oncol. 2010; 28(5):788-92. doi: 10.1200/JCO.2009.24.1315. Epub 2010 Jan 11. PMID: 20065186; PMCID: PMC2834395.

32. Rakha E, Wong SC, Soomro I, Chaudry Z, Sharma A, Deen $\mathrm{S}$, et al. Clinical outcome of atypical endometrial 
hyperplasia diagnosed on an endometrial biopsy: institutional experience and review of literature. Am J Surg Pathol. 2012; 36(11):1683-90. doi: 10.1097/ PAS.0b013e31825dd4ff. PMID: 23073327.

33. Whyte JS, Gurney EP, Curtin JP, Blank SV. Lymph node dissection in the surgical management of atypical endometrial hyperplasia. Am J Obstet Gynecol. 2010; 202(2):176.e1-4. doi: 10.1016/j.ajog.2009.10.855. Epub 2009 Dec 21. PMID: 20022313.

34. Taşkın S, Kan ö, Dai ö, Taşkın EA, Koyuncu K, Alkılıç A, et al. Lymph node dissection in atypical endometrial hyperplasia. J Turk Ger Gynecol Assoc. 2017; 18 (3): 127-132. doi: 10.4274/jtgga.2017.0043.

35. Giede KC, Yen TW, Chibbar R, Pierson RA. Significance of concurrent endometrial cancer in women with a preoperative diagnosis of atypical endometrial hyperplasia. J Obstet Gynaecol Can. 2008; 30(10):896-901. doi: 10.1016/S1701-2163(16)329693. PMID: 19038073; PMCID: PMC2891955.

36. Vilos GA, Oraif A, Vilos AG, Ettler H, Edris F, AbuRafea B. Long-term clinical outcomes following resectoscopic endometrial ablation of non-atypical endometrial hyperplasia in women with abnormal uterine bleeding. J Minim Invasive Gynecol. 2015; 22(1):66-77. doi: 10.1016/j.jmig.2014.07.009. Epub 2014 Jul 15. PMID: 25048567.

37. TrimbleCL, MethodM,LeitaoM,LuK,IoffeO,Hampton $\mathrm{M}$, et al.; Society of Gynecologic Oncology Clinical Practice Committee. Management of endometrial precancers. Obstet Gynecol. 2012; 120(5):11601175. doi: 10.1097/aog.0b013e31826bb121. PMID: 23090535; PMCID: PMC3800154.

38. Ghezzi F, Uccella S, Cromi A, Siesto G, Serati M, Bogani G, et al. Postoperative pain after laparoscopic and vaginal hysterectomy for benign gynecologic disease: a randomized trial. Am J Obstet Gynecol. 2010; 203(2):118.e1-8. doi: 10.1016/j.ajog.2010.04.026. Epub 2010 Jun 3. PMID: 20522410.

39. Ghezzi F, Uccella S, Cromi A, Bogani G, Robba $\mathrm{C}$, Serati $\mathrm{M}$, et al. Lymphoceles, lymphorrhea, and lymphedema after laparoscopic and open endometrial cancer staging. Ann Surg Oncol. 2012; 19(1):259-267. doi: 10.1245/s10434-011-1854-5. Epub 2011 Jun 22. PMID: 21695563.

40. American College of Obstetricians and Gynecologists. ACOG Committee Opinion No. 388 November 2007: supracervical hysterectomy. Obstet Gynecol. 2007; 110(5):1215-1217. doi: 10.1097/01. AOG.0000291564.32956.de. PMID: 17978148.
41. Boyraz G, Başaran D, Salman MC, Özgül N, Yüce K. Does Preoperative Diagnosis of Endometrial Hyperplasia Necessitate Intraoperative Frozen Section Consultation? Balkan Med J. 2016; 33(6):657-661. doi: 10.5152/balkanmedj.2016.151232. Epub 2016 Nov 1. PMID: 27994920; PMCID: PMC5156449.

42. Indermaur MD, Shoup B, Tebes S, Lancaster JM. The accuracy of frozen pathology at time of hysterectomy in patients with complex atypical hyperplasia on preoperative biopsy. Am J Obstet Gynecol. 2007; 196(5):e40-2. doi: 10.1016/j.ajog.2006.10.886. PMID: 17466676.

43. Morotti M, Menada MV, Moioli M, Sala P, Maffeo I, Abete L, et al. Frozen section pathology at time of hysterectomy accurately predicts endometrial cancer in patients with preoperative diagnosis of atypical endometrial hyperplasia. Gynecol Oncol. 2012; 125(3):536-40. doi: 10.1016/j.ygyno.2012.02.011. Epub 2012 Feb 21. PMID: 22361087.

44. Stephan JM, Hansen J, Samuelson M, McDonald M, Chin Y, Bender D, et al. Intra-operative frozen section results reliably predict final pathology in endometrial cancer. Gynecol Oncol. 2014; 133(3):499-505. doi: 10.1016/j.ygyno.2014.03.569. Epub 2014 Apr 1. PMID: 24699308.

45. Attard Montalto S, Coutts M, Devaja O, Summers J, Jyothirmayi R, Papadopoulos A. Accuracy of frozen section diagnosis at surgery in pre- malignant and malignant lesions of the endometrium. Eur J Gynaecol Oncol. 2008; 29(5):435-440.

46. Oz M, Ozgu E, Korkmaz E, Bayramoglu H, Erkaya S, Gungor T. Utility of frozen section pathology with endometrial pre-malignant lesions. Asian Pac J Cancer Prev. 2014; 15(15):6053-6057. doi: 10.7314/ apjcp.2014.15.15.6053. PMID: 25124573.

47. Salman MC, Usubutun A, Dogan NU, Yuce K. The accuracy of frozen section analysis at hysterectomy in patients with atypical endometrial hyperplasia. Clin Exp Obstet Gynecol. 2009; 36(1):31-34. PMID: 19400415.

48. Oda K, Koga K, Hirata T, Maruyama M, Ikemura $\mathrm{M}$, Matsumoto Y, et al. Risk of endometrial cancer in patients with a preoperative diagnosis of atypical endometrial hyperplasia treated with total laparoscopic hysterectomy. Gynecol Minim Invasive Ther. 2016; 5 (2016): 69-73. https://doi.org/10.1016/j. gmit.2016.02.001.

49. Beutler HK, Dockerty MB, Randall LM. Precancerous lesions of the endometrium. Am J Obstet Gynecol. 
1963; 86:433-43. doi: 10.1016/0002-9378(63)90167-4. PMID: 13967897.

50. Campbell PE, Barter RA. The significance of a typical endometrial hyperplasia. J Obstet Gynaecol Br Commonw. 1961; 68:668-72. doi: 10.1111/j.14710528.1961.tb02789.x. PMID: 13690268.

51. Gore H, Hertig AT. Carcinoma in situ of the endometrium. Am J Obstet Gynecol. 1966; 94(1):134155. doi: 10.1016/0002-9378(66)90391-7. PMID: 5321298.

52. Urban R, Reed S. Management of endometrial hyperplasia [Internet]. Waltham: UpToDate; 2020 [consultado 23 de abril de 2020]. Disponible en: https://www.uptodate.com/contents/management-ofendometrial-hyperplasia.

53. Gallos ID, Yap J, Rajkhowa M, Luesley DM, Coomarasamy A, Gupta JK. Regression, relapse, and live birth rates with fertility-sparing therapy for endometrial cancer and atypical complex endometrial hyperplasia: a systematic review and metaanalysis. Am J Obstet Gynecol. 2012; 207(4):266.e1-12. doi: 10.1016/j.ajog.2012.08.011. Epub 2012 Aug 10. PMID: 23021687.

54. Gallos ID, Shehmar M, Thangaratinam S, Papapostolou TK, Coomarasamy A, Gupta JK. Oral progestogens vs levonorgestrel-releasing intrauterine system for endometrial hyperplasia: a systematic review and metaanalysis. Am J Obstet Gynecol. 2010; 203(6):547. e1-10. doi: 10.1016/j.ajog.2010.07.037. PMID: 20934679.

55. Randall TC, Kurman RJ. Progestin treatment of atypical hyperplasia and well-differentiated carcinoma of the endometrium in women under age 40. Obstet Gynecol. 1997; 90(3):434-340. doi: 10.1016/s00297844(97)00297-4. PMID: 9277658.

56. Wheeler DT, Bristow RE, Kurman RJ. Histologic alterations in endometrial hyperplasia and welldifferentiated carcinoma treated with progestins. Am J Surg Pathol. 2007; 31(7):988-98. doi: 10.1097/ PAS.0b013e31802d68ce. PMID: 17592264.
57. Shan W, Wang C, Zhang Z, Gu C, Ning C, Luo X, et al. Conservative therapy with metformin plus megestrol acetate for endometrial atypical hyperplasia. J Gynecol Oncol. 2014; 25(3):214-220. doi: 10.3802/ jgo.2014.25.3.214. Epub 2014 Jul 3. PMID: 25045434; PMCID: PMC4102740.

58. Mitsuhashi A, Sato Y, Kiyokawa T, Koshizaka M, Hanaoka H, Shozu M. Phase II study of medroxyprogesterone acetate plus metformin as a fertility-sparing treatment for atypical endometrial hyperplasia and endometrial cancer. Ann Oncol. 2016; 27(2):262-266. doi: 10.1093/annonc/mdv539. Epub 2015 Nov 16. PMID: 26578736.

59. Gunderson CC, Fader AN, Carson KA, Bristow RE. Oncologic and reproductive outcomes with progestin therapy in women with endometrial hyperplasia and grade 1 adenocarcinoma: a systematic review. Gynecol Oncol. 2012; 125(2):477-482. doi: 10.1016/j. ygyno.2012.01.003. Epub 2012 Jan 11. PMID: 22245711.

60. Gallos ID, Krishan P, Shehmar M, Ganesan R, Gupta JK. Relapse of endometrial hyperplasia after conservative treatment: a cohort study with long-term follow-up. Hum Reprod. 2013; 28(5):1231-1236. doi: 10.1093/ humrep/det049. Epub 2013 Mar 6. PMID: 23466671.

61. Ørbo A, Arnes M, Vereide AB, Straume B. Relapse risk of endometrial hyperplasia after treatment with the levonorgestrel-impregnated intrauterine system or oral progestogens. BJOG. 2016; 123(9):1512-1519. doi: 10.1111/1471-0528.13763. Epub 2015 Dec 2. PMID: 26630538; PMCID: PMC5215722.

62. Park JY, Lee SH, Seong SJ, Kim DY, Kim TJ, Kim JW, et al. Progestin re-treatment in patients with recurrent endometrial adenocarcinoma after successful fertilitysparing management using progestin. Gynecol Oncol. 2013; 129(1):7-11. doi: 10.1016/j.ygyno.2012.12.037. Epub 2012 Dec 30. PMID: 23283299.

Recibido 11 de mayo de 2020 Aprobado 18 de agosto de 2020 